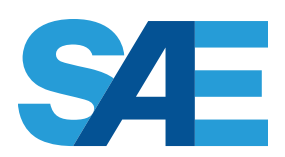

INTERNATIONAL

\title{
Techniques for Aerodynamic Analysis of Cornering Vehicles
}

\section{James Keogh and Tracie Barber}

UNSW Australia

\section{Sammy Diasinos}

Macquarie University

\section{Graham Doig}

California Polytechnic State University

CITATION: Keogh, J., Barber, T., Diasinos, S., and Doig, G., "Techniques for Aerodynamic Analysis of Cornering Vehicles," SAE Technical Paper 2015-01-0022, 2015, doi:10.4271/2015-01-0022.

\begin{abstract}
When a vehicle travels through a corner it can experience a significant change in aerodynamic performance due to the curved path of its motion. The yaw angle of the flow will vary along its length and the relative velocity of the flow will increase with distance from the central axis of its rotation. Aerodynamic analysis of vehicles in the cornering condition is an important design parameter, particularly in motorsport. Most racing-cars are designed to produce downforce that will compromise straight-line speed to allow large gains to be made in the corners. Despite the cornering condition being important, aerodynamicists are restricted in their ability to replicate the condition experimentally. Whirling arms, rotary rigs, curved test sections and bent wind tunnel models are experimental techniques capable of replicating some aspects of the cornering condition, but are all compromised solutions.
\end{abstract}

Numerical simulation is not limited in the same way and permits investigation into the condition. However, cornering introduces significant change to the flowfield and this must be accommodated for in several ways. Boundary conditions are required to be adapted to allow for the curved flow occurring within a non-inertial reference frame. In addition, drag begins to act in a curved path and variation in Re occurs within the domain. Results highlight the importance of using correct analysis techniques when evaluating aerodynamic performance for cornering vehicles.

\section{Introduction}

Aerodynamic performance through a corner is a critical design consideration for a number of applications. Highly maneuverable aircraft and racing vehicles are some examples. When a vehicle travels through a corner it can experience a significant change in aerodynamic performance due to its curved path of motion. In the reference frame of the body, the flow is observed to pass over the body following the same curve.

Figure 1 shows the freestream flow conditions for a vehicle in the steady-state cornering condition. The car itself is described as having a constant angular velocity about an external point. The velocity of the flow relative to the car will increase with distance from this central axis of rotation. In addition the angle (effectively yaw) of the flow will vary along the length of the car. In the specific condition shown it can be observed that the front and rear have opposite angles of yaw. The condition shown has a rear slip angle which is equal to the steering angle, minus slip, at the front. The real-world angle of the vehicle through a corner will vary with different cars and driving styles [1]. The static pressure will remain the same throughout the domain. It can be seen that, particularly for a small radius corner, the condition is a significant departure from freestream conditions in a straight line, or a constant angle of yaw.

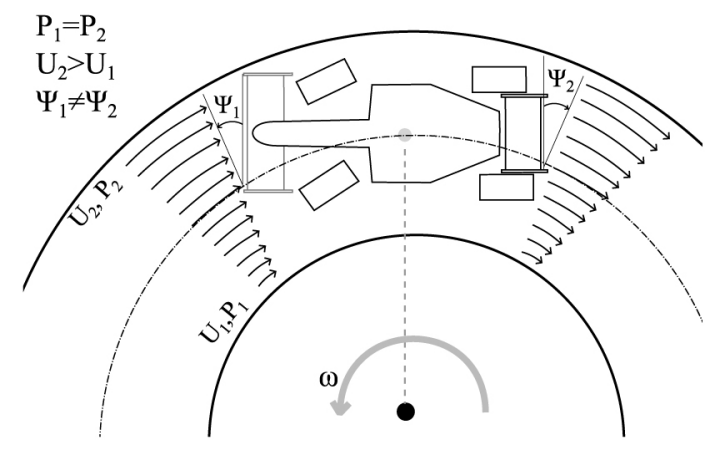

Figure 1. The steady-state cornering flow conditions. 
For most racing-cars the aerodynamics are designed in a way that ultimately compromises straight-line top speed but allows large gains to be made in the corners $[\underline{2}, \underline{3}, \underline{4}]$. With the addition of downforce acting on the tyres, the effective adhesion increases the lateral acceleration that is able to be sustained through a corner. From the 1960 's to 1990 's the maximum lateral acceleration for racing-cars increased from approximately 1.2 times gravitational acceleration to close to four times; with the contribution from improved tyre technology only being small []].

Despite aerodynamic performance being most critical while cornering, designs will typically be evaluated in the straight-line condition. This is largely due to the wind-tunnel remaining the primary tool for aerodynamic development. Industry is aware of the limitations of these methodologies [5]. At present the true condition has not been achieved experimentally in the public domain, leaving numerical simulation as the preferred option for this type of analysis.

A previous study considered two variations of a simple passenger car geometry, analysed numerically and on road, to examine the aerodynamic effects experienced during sinusoidal motion $[\underline{6}, \underline{7}]$. The modified vehicle geometry differed from the original as it had a reduced space around the wheel in the wheel-well. The static pressure was measured using surface static pressure disk probes, while total pressure utilised Kiel probes positioned in the front wheel wake. The difficulties in gaining meaningful results from the driving vehicle were acknowledged, and all on-road forces were reported as estimates based on integration of the surface pressure measurements, calibrated using wind tunnel data of the vehicle in the yaw condition highlighting the difficulties of obtaining accurate experimental results.

In a separate study an open-wheel racing car was numerically analysed through three specific corners of the Fuji Speed Way Circuit []]. The study presented the variation in aerodynamic forces that occurred due to the change in conditions and highlighted changes in yawing moment and side force through corners. Further detail on the flow structures causing these effects was not included.

While the capabilities of numerical simulation are constantly improving, a known weakness is their ability to correctly predict sensitivities to a change in conditions [ $\underline{5}$ ]. Ride height sweeps and yaw (or crosswind) will still be preferably tested in the wind tunnel, rather than simulated. Currently aerodynamicists must use numerical simulation for the cornering condition, while validating results with experimental data for the straight-line, or yawed condition.

\section{Experimental Methods for Cornering}

Currently there is no experimental technique for simulating cornering in a controlled environment. It is a condition that can be analysed on a track or road but this is limited by the variability of real-world conditions and the difficulty of obtaining data from an actual vehicle [7].

The most immediately obvious solution is to create a track within a controlled environment, where an instrumented model could be propelled around. In the large majority of cases this isn't feasible due to the significant space requirements and inevitable cost. Flow settling time between runs, instrumentation and the time requirement to test variables such as ride height may be some of the limitations. To date such facility types have only been used for straight-line testing [9]. Placing a model in different combinations yaw is believed by some to be the best method currently available for experimentally representing cornering for vehicles [ $\underline{5}]$.

There have been, and are, a number of experimental rigs and techniques used that can come close to producing the curved flow that a vehicle will experience through a corner. Unfortunately they are all limited in different ways.

\section{Whirling Arm}

The whirling arm preceded the wind tunnel. It operates by moving the model through the air about a central pivot, shown in Fig. 2 . The first was built by an English mathematician Benjamin Robins in 1746 [10]. His whirling arm was driven by a falling weight and had arm length of $1.2 \mathrm{~m}$ with a maximum tip tangential velocity of approximately $1 \mathrm{~m} / \mathrm{s}$. Further experiments were conducted by Sir George Cayley with an improved design that permitted higher speed. He considered various aerofoil shapes and obtained lift and drag data.

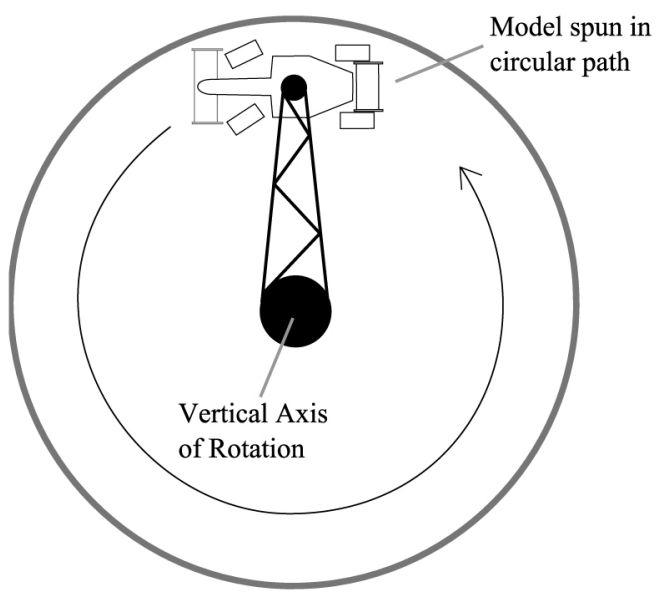

Figure 2. The motion of a model in a whirling arm facility.

For testing vehicles travelling through a corner the design has a lot of desirable characteristics. The motion of the model past the fixed ground would mean a rolling road is no longer required, and if the freestream flow were stationary as the model passed through it, then the cornering condition would be achieved. However, the flaws of this design were apparent to the first users. The spinning arm meant the model would always be travelling through its own wake and this lead to a high level of turbulence and swirl in the flow. A highly turbulent, whirlpool-like flow environment would typically result due to the model's motion.

Despite the known issues, the National Physics Laboratory (NPL), in the UK, built a new whirling arm in 1908 [11]. The rig was driven by an electric motor with a design radius of $9 \mathrm{~m}$. It was initially intended for the testing of airships (the most promising form of air travel at that stage). Three re-designs were undertaken by the NPL from 1908-1942 before the rig was transferred to Cranfield University, shown in Fig. 3 . Modifications attempted to reduce the swirl in the 
flow through the use of baffles and removing any unnecessary bulky measurement devices in the test section. The swirl itself was dependent on the drag of the model and strut in use, but varied from $7.5-22.5 \%$ across various configurations. Experiments considering a wing in ground effect, hovercraft and aircraft were conducted, but very few results were published, largely due to the issues with flow quality $[\underline{11}, \underline{12}, \underline{13}]$.

More recently a whirling arm was used for validation of theoretical calculations of regarding a wings dihedral angle [14]. The experiments utilised a $3.5 \mathrm{~m}$ radius whirling arm with a tip velocity of $7 \mathrm{~m} / \mathrm{s}$. The facility utilised was initially intended for the calibration of anemometers, and had to be adapted for the experiments. There was no report of the flow quality and very limited results.

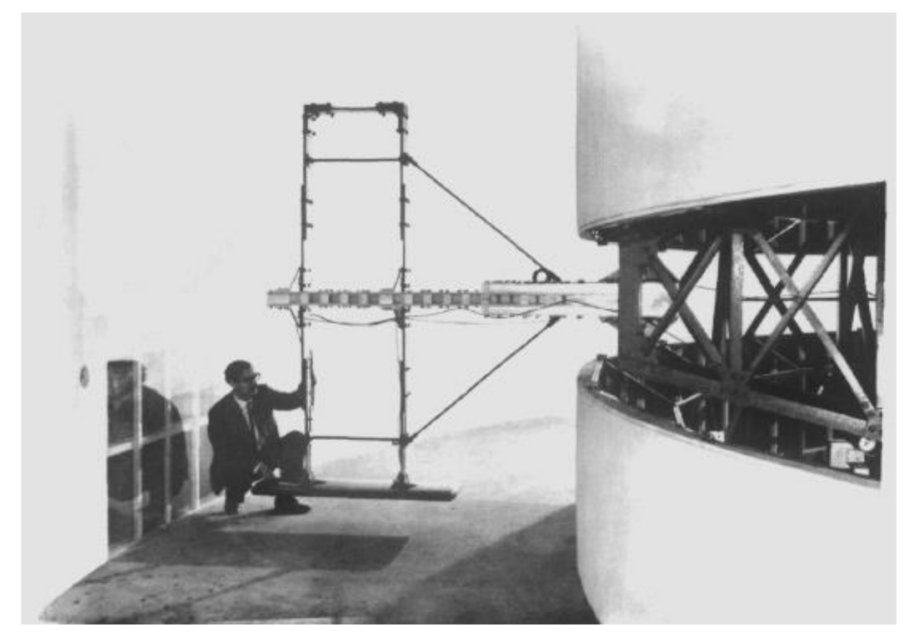

Figure 3. The Cranfield whirling arm facility, showing the model positioned in the test section.

\section{Rotary Rig}

With aircraft becoming more maneuverable, testing of dynamic stability parameters became important [15]. As a result different types of rotary rig were developed for use in wind tunnels. These are continuing to become more advanced and offer multiple degrees of freedom [16]. The most similar type of motion to a vehicle cornering is coning - this is where the model will be rotated about an axis parallel to the freestream direction. The resultant path then becomes helical as is shown in Fig. 4. This overcomes the issue of the model running through its own wake.

Sting interference with the flow and changing proximity to the test section walls are some common issues. An adaption to automotive aerodynamic testing would be difficult. Large or open test sections are already required to allow for the large blockage caused by a bluff-body. This type of rig would then require either a much larger test-section or the use of a smaller model. A further issue would be representation of the ground plane. The model could be designed as attached to a fixed ground plane, or be in motion with a moving ground, but both options would become exceedingly complex and result in an increased blockage ratio. a)

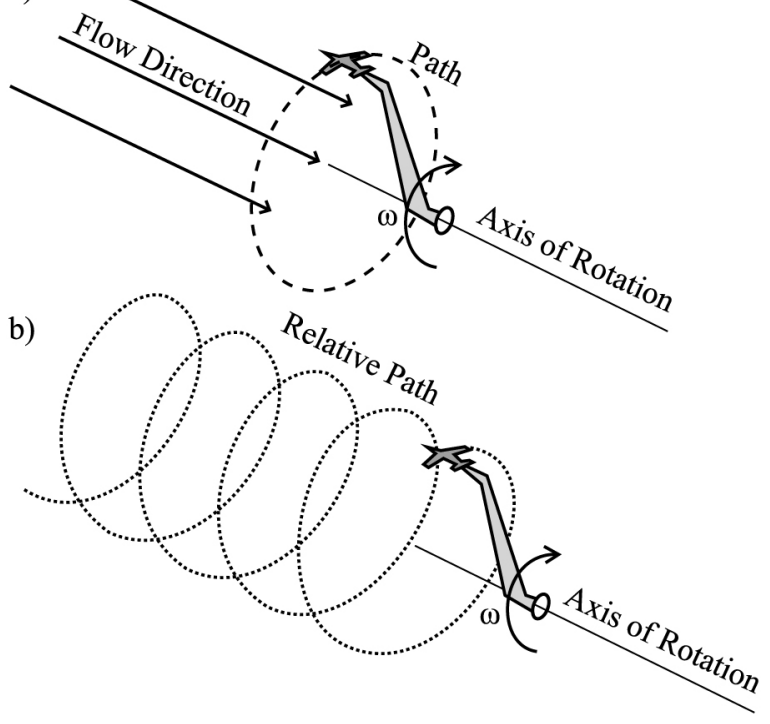

Figure 4. Steady-state coning motion of a wind tunnel model, as produced by a rotary rig: a) In the absolute reference frame b) In the reference frame of the model

Some dynamic motions are achieved with an overhead traverse in the more advanced automotive wind tunnels $[\underline{17}, \underline{18}]$, but these have very little scope for significant curvatures to be achieved.

\section{Curved Test Section}

A curved test section is another method that has been used in order to force curved flow over a model. In 1939 the Langley Research Centre built a wind tunnel designed to be capable of testing an aircraft in rolling, pitching and yawing. The Langley Stability Tunnel used interchangeable test-sections, one which was capable of curving the walls [19], as is shown in Fig. 5.

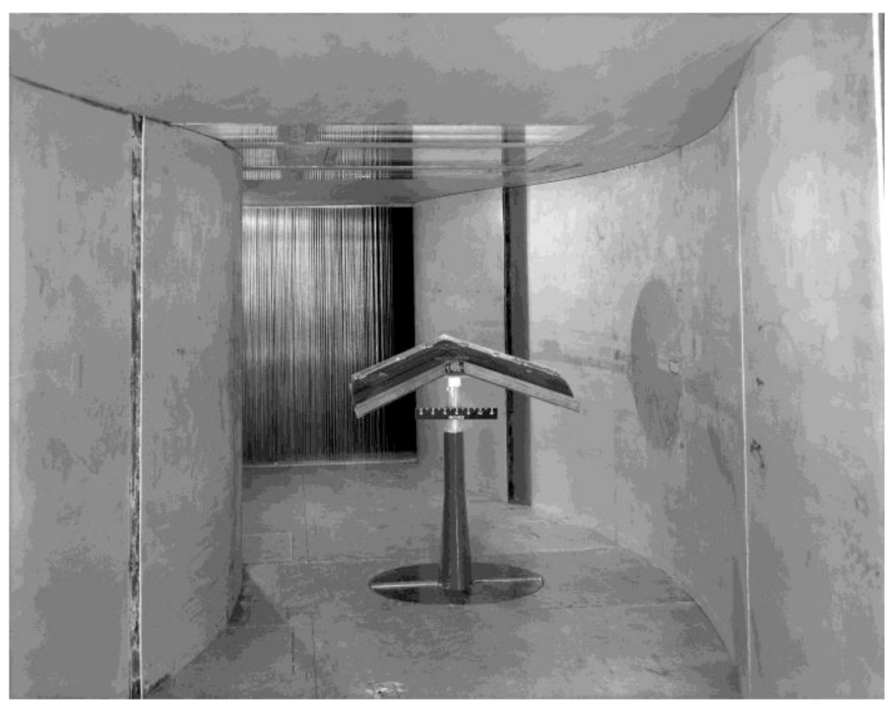

Figure 5. A view inside the Langley Stability Tunnel curved test section [16].

Forcing flow to follow the shape of a curved test section ultimately compromises the flow quality. For the cornering condition, shown in Fig. 1, the outer radius is larger and the velocity is higher, the converse is true for the inner radius. A large body of work exists for flows in curved ducts and the shape results in a suction and pressure surface [20]. The outer curve is a concavity which causes 
deceleration of the flow and an increase in pressure, the inner surface is convex which accelerates the flow and decelerates the pressure. The net result is a velocity profile that is near the exact opposite of what would be desired, and a static pressure profile across the test section. These effects then increase in severity as the radius of curvature is decreased.

\section{Bent Model}

A different approach specifically considers the change in angle of the flow over the body through a corner, as is shown in Fig. 6. An idea that has been proposed in a number of studies $[5,21,22]$ is using a model that is curved, relative to the straight freestream flow, rather than having curved freestream flow with a straight model.

An obvious first issue is the feasibility of physically constructing such a model for a car. The wheels have to be curved, yet they will also be rotating - requiring their shape to change as they spin. A new model is also required for each type of corner.

Furthermore the curved shape causes one side of the model to become longer than the other. The side that extends in length is the side representative of the inside of the corner, as can be seen in Fig. 6. Similar to a flaw of the curved test section this then results in higher local Reynolds numbers occurring on the side closest to the inside of the corner, and lower local Reynolds numbers on the outside. The opposite of what occurs in reality.

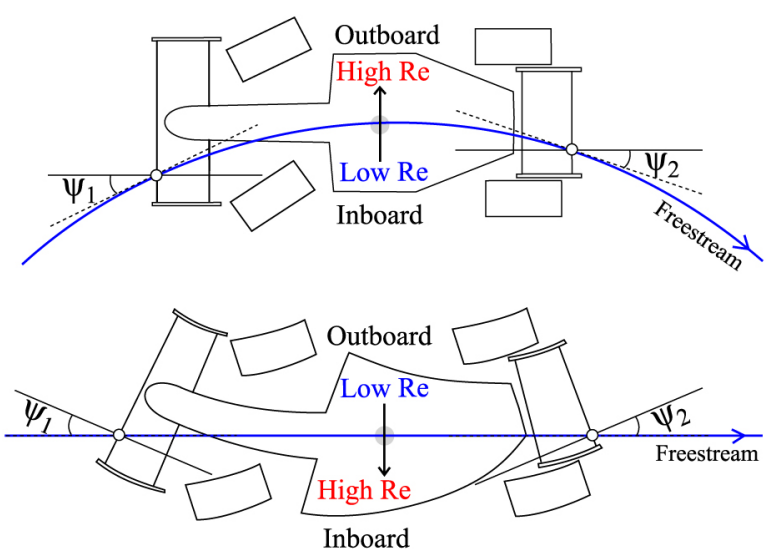

Figure 6. An example of the wind tunnel model bending method used to attempt replicate the cornering condition for a left-hand turn. The incorrect change in local Reynolds due to model bending is indicated.

\section{Numerical Methods for Cornering}

Numerical simulation is the preferred method for cornering aerodynamics analysis $[\underline{5}, \underline{6}, \underline{7}, \underline{8}]$. In terms of the boundary conditions and the structure of the domain there has been some variation amongst studies. In most instances the domain is defined as a non-inertial reference frame. Motion is defined by prescribing an angular velocity about a point external to the domain, as is shown in Fig. 7. This radius and the exact position of the point are determined by the corner being considered and the dynamics of the vehicle.

In one study, dynamic motion of the vehicle through the mesh was preferred, to avoid the additional computational expense required for Coriolis terms and acceleration terms in a non-inertial reference frame []․ This was coupled with sides of the domain varying between inlet and outlet conditions as required. The efficiency of this method is solver and mesh dependent. In the present study the preferred method has been the use of a non-inertial reference frame, where an angular velocity is prescribed about a point external to the domain.

\section{Boundary Conditions}

As the freestream flow travels in a curved path this introduces additional considerations when constructing the numerical domain and determining boundary conditions. With curvature of the freestream flow the domain can be constructed to also be curved, as is shown in Fig. 7a). However, in a practical sense this would then limit the use of that mesh to just one specific cornering condition, and wouldn't permit any form of dynamic simulation.

Tsubokura et. al [ $\underline{6}]$ and Nara et. al [ $\underline{8}]$ both opted for the use of a rectangular domain using modified inlet and outlet boundary conditions along the domain wall to achieve the desired flowfield, as shown in Fig. 7b). This method permits the evaluation of multiple corners utilizing the same mesh, and also allows dynamic simulations incorporating variable curvatures and directions.

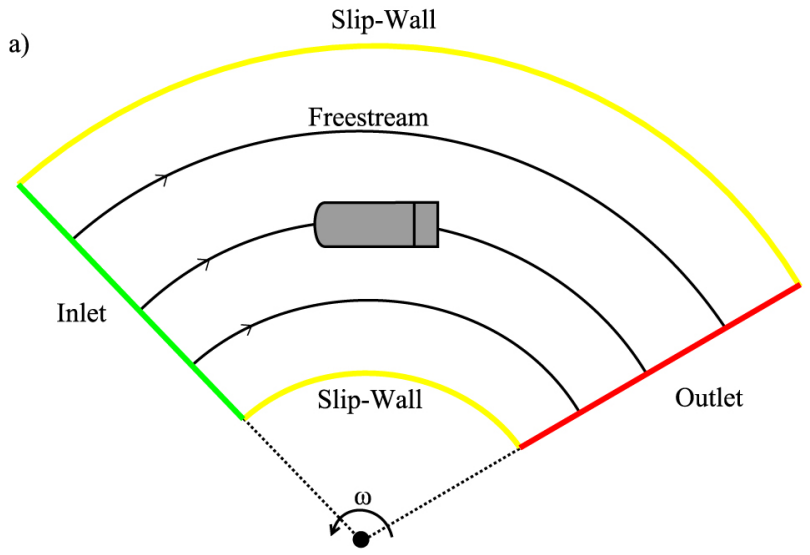

b)

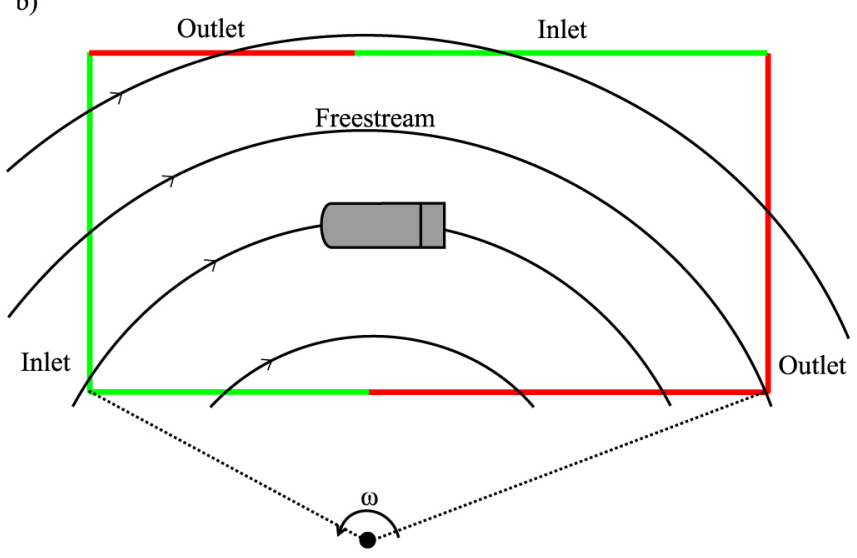

Figure 7. a) An example implementation of a curved domain, and b) rectangular domain for analysis of a steady-state corner.

\section{Aerodynamic Force Analysis for Cornering}

Aerodynamic drag, by definition, is the resistance due to the freestream flow in the direction of a vehicle's motion. Therefore as a vehicle travels in the curved path of a corner, drag itself also acts 
following a curved path, as is shown in Fig. 8. This then fundamentally changes the way it should be calculated. Instead of being a force which acts in a straight-line, the angle changes along the length of a body with the freestream. More simply it becomes proportional to the moment acting about the centre of rotation of the vehicle's path. The direction of lift remains unaffected as it is parallel to the axis of rotation. Side force will also continue to act in the same direction, but will differ slightly from the radial force. All moments will continue to act in the normal direction.

The example of a generic car shape is utilised to demonstrate the difference between drag, and the force coefficient in the $\mathrm{x}$-direction.

Time-averaged results are compared for an Ahmed Body [23] , shown in Fig. 8, with a rear backlight angle of $25^{\circ}$ travelling through steady-state corners with radii (R) from 5 to 20 car lengths (L), shown in Fig. 9. Results were calculated using a commercial finite volume solver, adopting the Detached Eddy Simulation (DES) technique, with a Realizable k- $\varepsilon$ wall model. The tangential velocity was constant at $25 \mathrm{~m} / \mathrm{s}$ at the centre of the vehicle, giving a lengthbased Re of $1.7 \times 10^{6}$ at that location, for all simulations. Further details on the method can be found in Keogh et. al [24].

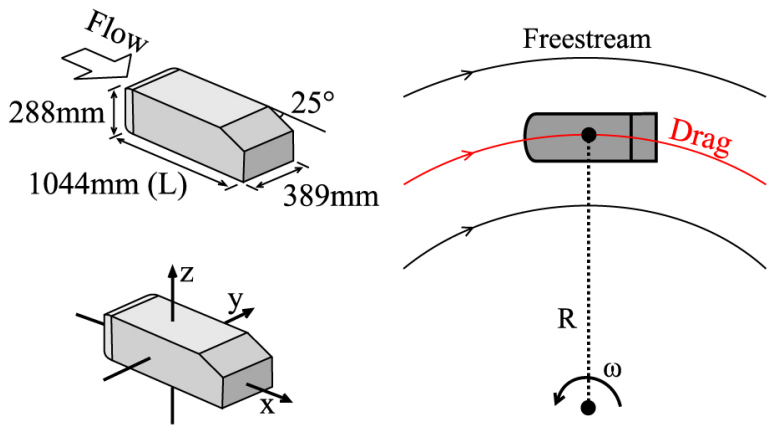

Figure 8. Dimensions of the Ahmed Body geometry and the type of motion considered for the numerical simulations [24].

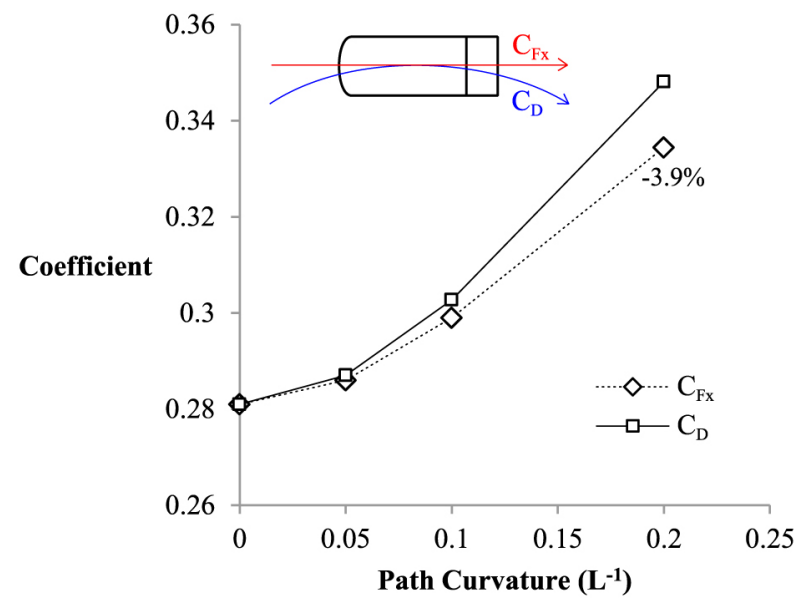

Figure 9. Change in drag and force in the x-direction for the Ahmed Body with increasing curvature in the path of motion.

A second issue occurs when looking to normalise forces for a uniform measure of aerodynamic performance. From Fig. 1 it can be observed that the freestream velocity will increase with distance from central axis of rotation. This produces local changes in Reynolds number at different point over the vehicle. Dependent on where the reference velocity is calculated this can lead to misconstrued aerodynamic performance.
A comparison between using a constant velocity value (equal to freestream at the vehicle's centre) and using the freestream velocity distribution is shown in Table 1. The two techniques are compared for a $5 \mathrm{~L}$ radius corner. The calculation of these forces from pressure and shear data over the surface is detailed in the Appendix.

Table 1. The change in calculated drag value for the Ahmed Body in the cornering condition; normalized against the velocity distribution $\left(\mathrm{C}_{\mathrm{DC}}\right)$ and reference velocity value $\left(\mathrm{C}_{\mathrm{D}}\right)$.

\begin{tabular}{lccc}
\hline Component & $\mathbf{C}_{\mathbf{D}}$ & $\mathbf{C}_{\mathbf{D C}}$ & \% Difference \\
Pressure & 0.3010 & 0.3009 & 0.07 \\
Viscous & 0.0564 & 0.0564 & 0.03 \\
Overall & 0.3574 & 0.3573 & 0.05 \\
\hline
\end{tabular}

Results demonstrate that, in this particular instance, the use of a constant value makes only a very small change to the true force coefficient, and can be regarded as a sufficient approximation. The importance of being able to easily translate from a force coefficient to a force value is also a practical reason for using the single value.

\section{Summary}

The cornering condition can have a significant effect on aerodynamic performance of vehicles but is unable to be represented experimentally. Experimental solutions developed for dynamic motion of aircraft are not readily adaptable for automotive bodies. The close ground proximity, high blockage ratio and the specific type of motion all add complexity which increases the difficulty of achieving the required flow conditions.

Fortunately numerical simulation permits investigation into the condition. However aerodynamicists must remain aware of the change affected by this condition. Numerical simulation must accommodate curved flow occurring within a non-inertial reference frame, and this requires additional considerations when constructing such models. Due to the motion, drag begins to act in a curved path and variation in Re occurs within the domain. Results highlight the importance of adopting the correct analysis techniques when evaluating aerodynamic performance for cornering vehicles.

\section{References}

1. Milliken, W., and Milliken, D., "Race Car Vehicle Dynamics" (Warrendale, Society of Automotive Engineers, Inc., 1994), ISBN 978-1-56091-526-3.

2. Dominy RG. Aerodynamics of Grand Prix Car Proc. Inst. Mech. Eng. 1992; 206:267-274.

3. Katz J. Aerodynamics of Race Cars. Annu. Rev. Fluid Mech. 2006; 38:27-63. doi:10.1146/annurev.fluid.38.050304.092016.

4. Zhang X, Toet W and Zerihan J. Ground Effect Aerodynamics of Race Cars. Appl. Mech. Rev. 2006; 59:33-49. doi: $10.1115 / 1.2110263$.

5. Toet W. Aerodynamics and aerodynamic research in Formula 1. Aeronaut. J. 2013; 117(1187):1-26. 
6. Tsubokura, M., Ikawa, Y., Nakashima, T., Okada, Y. et al., "Unsteady Vehicle Aerodynamics during a Dynamic Steering Action: 2nd Report, Numerical Analysis," SAE Int. J. Passeng. Cars - Mech. Syst. 5(1):340-357, 2012, doi:10.4271/2012-01$\underline{0448}$.

7. Okada, Y., Nouzawa, T., Okamoto, S., Fujita, T. et al., "Unsteady Vehicle Aerodynamics during a Dynamic Steering Action: 1st Report, On-Road Analysis," SAE Technical Paper 2012-01-0446, 2012, doi:10.4271/2012-01-0446.

8. Nara, K., Tsubokura, M., Ikeda, J., Fasel, U., et. al "Numerical Analysis of Unsteady Aerodynamics of Formula Car during Dynamic Cornering Motion," 32 $2^{\text {nd }}$ AIAA Applied Aerodynamics Conference, June 2014, Atlanta, GA, USA

9. Curtis, H., Putman, W. and Traybar, J., "The Princeton Dynamic Model Track," Aerodynamic Testing Conference, Washington D.C., USA, 1964 doi:10.2514/6.1964-1104.

10. Baals, D. and Corliss, W., Wind Tunnels of NASA, NASA accessed October 30, 2014. http://www.hq.nasa.gov/pao/ History/SP-440/contents.htm

11. Mulkens, M. and Ormerod, A., "Steady-State Experiments for Measurements of Aerodynamic Stability Derivatives of a High Incidence Research Model Using the College of Aeronautics Whirling Arm," College of Aeronautics Report No. 9014, 1990

12. Llewelyn-Davies, M. "The Redesign of the College of Aeronautics Whirling Arm Facility", College of Aeronautics Report No. 8702, 1987

13. Kumar, P. "The College of Aeronautics Whirling Arm Initial Development Tests," CoA Note Aero. No. 174, 1967

14. Gili, P. and Battipede, M., "Experimental Validation of the Wing Dihedral Effect Using a Whirling Arm Experiment," J. Aircraft 2001; 38(6):1069-1075. doi: $10.2514 / 2.2874$

15. Ericsson, E., "Reflections Regarding Recent Rotary Rig Results," J. Aircraft 1987; 24(1): 25-30. doi:10.2514/3.45406

16. Pattison, J., Lowenberg, M. and Goman, M., "Multi-Degreeof-Freedom Wind-Tunnel Maneuver Rig for Dynamic Simulation and Aerodynamic Model Identification," J. Aircraft 2013;50(2):551-566. doi:10.2514/1.C031924

17. Aschwanden, P., Müller, J., Travaglio, G., and Schöning, T., "The Influence of Motion Aerodynamics on the Simulation of Vehicle Dynamics," SAE Int. J. Passeng. Cars - Mech. Syst. 1(1):545-551, 2009, doi:10.4271/2008-01-0657.

18. Duell, E., Kharazi, A., Muller, S., Ebeling, W. et al., "The BMW AVZ Wind Tunnel Center," SAE Technical Paper 2010-01-0118, 2010, doi:10.4271/2010-01-0118.

19. "Stability Tunnel," last modified July 8, 2014, http://crgis.ndc. nasa.gov/historic/Stability Tunnel

20. Berger, S., Talbot, L. and Yao, L., "Flow in Curved Pipes" Ann. Rev. Fluid Mech. 1983. 15:461-512

21. Gordes A. Process for simulating curved airflow on wheeled vehicles in fluid channels with a straight measuring section Patent No. EP1610111A2, Germany, 2005.

22. Gregory, P., Joubert, P. and Chong, M., Flow Over a Body of Revolution in a Steady Turn, Australia: DSTO Platforms Sciences Library, 2004
23. Ahmed, S., Ramm, G., and Faltin, G., "Some Salient Features Of The Time-Averaged Ground Vehicle Wake," SAE Technical Paper 840300, 1984, doi: $10.4271 / 840300$.

24. Keogh J, Doig G, Diasinos S, and Barber T, "Detached Eddy Simulation of the Cornering Aerodynamics of the Ahmed Reference Model" FISITA World Automotive Conference, Maastricht, the Netherlands, June 2014

\section{Contact Information}

James Keogh

School of Mechanical and Manufacturing Engineering

UNSW Australia

j_keogh@live.com.au

\section{Definitions/Abbreviations}

$\mathbf{C}_{\mathbf{D}}$ - coefficient of force in the direction aligned with freestream

$\mathbf{C}_{\mathbf{D c}}$ - coefficient of drag, corrected according to local freestream velocity magnitude

$\mathbf{C}_{\mathbf{F x}}$ - coefficient of force in the $\mathrm{x}$ direction

L - body length $1044 \mathrm{~mm}$

$\mathbf{R}$ - radius of curvature $m$

$\mathbf{U}_{\infty}$ - freestream velocity $\mathrm{ms}^{-1}$

$\mathbf{U}, \mathbf{V}, \mathbf{W}$ - velocity components in the $\mathrm{x}, \mathrm{y}$ and $\mathrm{z}$ directions $\mathrm{ms}^{-1}$

$\mathbf{x}, \mathbf{y}, \mathbf{z}$ - Cartesian right handed coordinates

$\boldsymbol{\kappa}$ - flow curvature $(1 / \mathrm{R}) \mathrm{m}^{-1}$

$\psi$ - angle of incidence about the z-axis 


\section{APPENDIX}

As the freestream flow assumes a curvature relative to the body, the assumption of drag acting linearly becomes incorrect. Analyzing a body following a curved path of motion introduces change in the way aerodynamic forces are determined. The drag force becomes proportional to the moment acting on the body in the same circular path. Lift continues to act in the vertical direction. The calculation of coefficients relative to a constant freestream value can become unsuitable when looking to establish a consistent aerodynamic performance coefficient.

The drag coefficient (for incompressible flow) can be calculated as shown below:

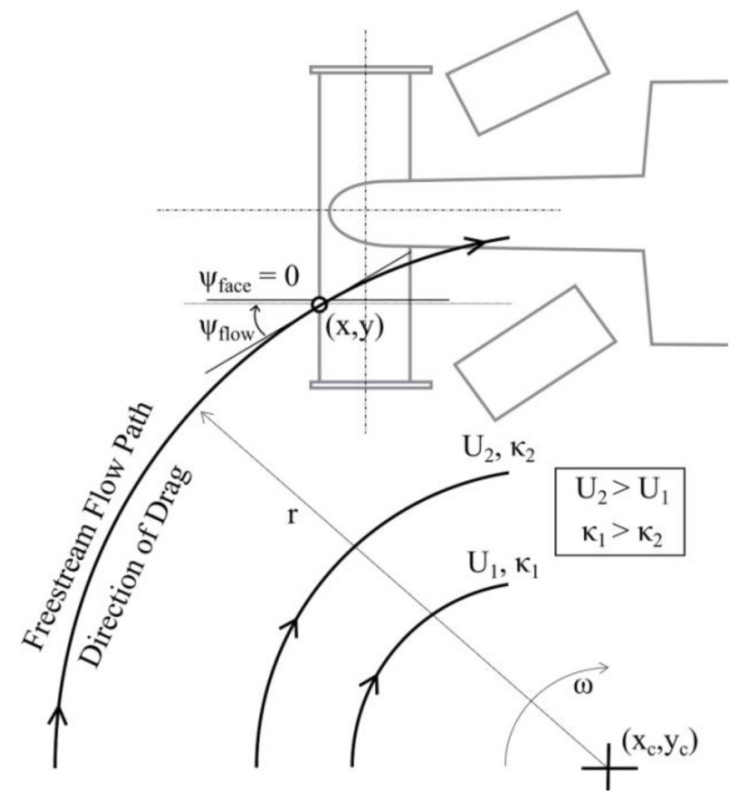

The velocity at any given point in the flow-field is described with respect to the instantaneous center of rotation shown in Fig. XX at $\left(x_{c}, y_{c}\right)$.

$$
U_{\infty}=\omega \times \sqrt{\left(y-y_{c}\right)^{2}+\left(x-x_{c}\right)^{2}}
$$

Thus the corrected pressure coefficient $\left(\mathrm{C}_{\mathrm{pc}}\right)$ is defined where $U_{\infty}$ now varies, and $\mathrm{C}_{\mathrm{p}}$ with respect to the constant reference velocity is calculated in the same way, but uses a fixed $U_{\infty}$ value defined at the geometric centre of the body.

$$
C_{p}=\frac{p}{0.5 \times \rho \times U_{\infty}^{2}}
$$

The angle of the freestream flow with respect to the $\mathrm{x}$-axis is given by:

$$
\psi_{\text {flow }}=\tan ^{-1}\left(\frac{x-x_{c}}{y-y_{c}}\right)
$$

The angle of the cell face is determined by the projected face area of the cell in the $\mathrm{x}\left(A_{f x}\right)$ and $\mathrm{y}\left(A_{f y}\right)$ directions :

$$
\psi_{f a c e}=\tan ^{-1}\left(\frac{A_{f x}}{A_{f y}}\right)
$$


Ultimately what then becomes of interest is the differential angle between the flow and the body at the given location:

$$
\psi_{p}=\psi_{\text {face }}-\psi_{\text {flow }}
$$

The total face area of the cell in the x-y plane is given by:

$$
A_{f x y}=\sqrt{\left(A_{f x}\right)^{2}+\left(A_{f y}\right)^{2}}
$$

The observed face area of a given cell with respect to the freestream flow will be given by:

$$
A_{f o}=A_{f x y} \times \sin (\psi)
$$

The pressure drag component then becomes, where $\mathrm{C}_{\mathrm{pc}}$ replaces $\mathrm{C}_{\mathrm{p}}$ for a correction allowing for freestream variation:

$$
C_{D P}=\frac{\sum\left(C_{p} \times A_{f_{0}}\right)}{b \times c}
$$

The coefficients of wall-shear in each direction $\left(\mathrm{C}_{\tau \mathrm{x}}, \mathrm{C}_{\tau \mathrm{y}}, \mathrm{C}_{\tau \mathrm{z}}\right)$ are similarly non-dimensionalised using a constant $U_{\infty}$ value. $\mathrm{C}_{\tau \mathrm{xc}}, \mathrm{C}_{\tau \mathrm{yc}}$, and $\mathrm{C}_{\tau z \mathrm{c}}$ can be substituted and are calculated using the freestream velocity distribution.

The face area tangential to the flow direction is given by:

$$
A_{f t}=\sqrt{A_{f z}^{2}+\left(A_{f y} \cos \psi_{f l o w}+A_{f x} \cos \psi_{\text {flow }}\right)^{2}}
$$

The planar shear coefficient gives the wall-shear coefficient acting in the plane tangential to the direction of passage:

$$
C_{\tau x y}=\sqrt{C_{\tau x}^{2}+C_{\tau y}^{2}}
$$

The angle at which the planar shear is acting is given by:

$$
\psi_{\text {shear }}=\tan ^{-1}\left(\begin{array}{l}
\tau_{y} \\
\tau_{x}
\end{array}\right)
$$

The angle between the direction the shear is acting and the freestream flow angle again becomes of most interest:

$$
\psi_{v}=\psi_{\text {flow }}-\psi_{\text {shear }}
$$

The wall shear coefficient acting tangential to the freestream direction is then given by:

$$
C_{\tau t}=C_{\tau x y} \cos \psi_{v}
$$


As a result the viscous drag coefficient then becomes:

$$
C_{D v}=\frac{\sum C_{\tau t} \times A_{f t}}{b \times c}
$$

The overall drag coefficient $\left(\mathrm{C}_{\mathrm{D}}\right)$ is simply the sum of the two components

$$
C_{D}=C_{D v}+C_{D p}
$$

The Engineering Meetings Board has approved this paper for publication. It has successfully completed SAE's peer review process under the supervision of the session organizer. The proces requires a minimum of three (3) reviews by industry experts.

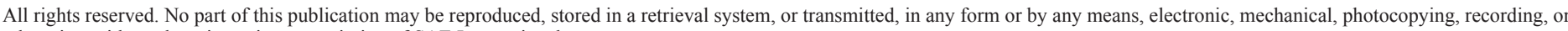
otherwise, without the prior written permission of SAE International.

Positions and opinions advanced in this paper are those of the author(s) and not necessarily those of SAE International. The author is solely responsible for the content of the paper.

ISSN 0148-7191

http://papers.sae.org/2015-01-0022 